\title{
Somatotopic principle of perineural implantation of stem cells in patients with brain injuries
}

\begin{abstract}
Background: Neuro destructive processes of any etiology are related to problematic and socially important diseases due to ineffective therapeutic strategy and need to search for new successful ways of treatment and rehabilitation of patient with cerebral infarctions and brain attacks
\end{abstract}

Aims: Authors plant overify hypothesis on viability of additional use of perineural implantation of autologous mesenchymal stem cells (MSC) in order to optimize standard therapy of patients with brain attacks. Such combined technology is aim datextra activation of brain plasticity mechanisms during development of neuro destructive processes.

Methods: The technique of MSC perineural migration to injured brain regions was experimentally verified on rats $(n=40)$ paying attention to somatotopic organization of cranial nerves.

This technique was clinically tested in pilot project. Phenotyping of autologous MSC from adipose tissue (AT) was performed in 23 patients with brain attacks. These 23 patients received standard treatment as per international guidelines together with three perineural implantations of autologous MSC from AT with 5-9days intervals. The other group of patients $(n=7)$ received only standard therapy as per international guidelines.

Results: Additional use of cell therapy resulted in more rapid and effective recovery of disordered neurological functions in all cases compared to those who received standard therapy. The phenomenon of abrupt recovery of neurological functions was established during first 24hours after each injection of autologous MSC. Cumulative recovery of functions progressed after each implantation.

Discussion and conclusion: Experimentally developed technique of perineural implantation of autologous MSC was successfully verified in clinical conditions in accordance with certified cell therapy guideline (The Ministry of Health of the Republic of Belarus) in combination with standard treatment of patients with cerebral infarctions. Cell therapy with autologous MSC from AT by means of perineural delivery to injured brain regions is the basis for activation of reparative potential of nerve tissue and progressive recovery of neurological functions in patients with cerebral infarctions.

Keywords: brain attack, stem cells, perineural migration, somatotopic principle, brain plasticity
Volume 8 Issue 5 - 2018

\author{
Shanko Yuri,' Navitskaya Valeria,' Zamaro \\ Alexandra, ${ }^{2}$ Zafranskaya Marina, ${ }^{3}$ Krivenko \\ Svetlana, ${ }^{4}$ Koulchitsky Stanislav, ${ }^{5}$ Takalchik- \\ Stukach Yulya, ${ }^{2}$ Smeyanovich Arnold,' \\ Nizheharodova Daria, ${ }^{3}$ Pashkevich Svetlana, ${ }^{2}$ \\ Dosina Margarita, ${ }^{2}$ Denisov Andrew, ${ }^{2}$ \\ Kulchitsky Vladimir ${ }^{2}$ \\ 'Republican Scientific and Practical Center of Neurology and \\ Neurosurgery, Belarus \\ ${ }^{2}$ Institute of Physiology, National Academy of Sciences, Belarus \\ ${ }^{3}$ Belarusian Medical Academy of Post-Graduate Education, \\ Belarus \\ ${ }^{49^{\text {th }}}$ City Clinical Hospital, Belarus \\ ${ }^{5}$ Liege University, Liege, Belgium
}

Correspondence: Vladimir Kulchitsky, Designation Scientific Director, University Institute of Physiology, NatlAcadSci, Address 28 Akademicheskaya Street, Minsk, Belarus, Tel +375 17 2842458,Email vladi@fizio.bas-net.by

Received: August 01, 2018 | Published: October 02, 2018
Abbreviations: SC, stem cells; MSC, mesenchymal stem cells; AT, adipose tissue; GDNF, glial cell derived neuro trophic factor [Homo sapiens (human)]; NGFR, nerve growth factor receptor; $\mathrm{i} / \mathrm{p}$, intraperitoneally

\section{Introduction}

Injuries, strokes and cerebral infarctions hold a specific place in the range of socially important diseases due to high level of mortality in all countries. ${ }^{1-3}$ Current situation initiates development to completely new methods of early diagnostics, therapy and rehabilitation in neuro destructive processes management. Small success was achieved on the way of increasing effectiveness of therapy at this stage. In particular, indications for SC use are broadened along with standard therapy of patients with neuro destructive processes. The search for "cerebral infarction, stroke, and stem cell" revealed 637 articles in PubMed on July 27, 2018. Promising results have been obtained at earlier stages of cell technologies combination with standard methods of therapy. ${ }^{4-7}$ However, the number of articles describing side effects of cell technologies also increased. ${ }^{8-11}$
Disadvantages of system injection of stem cells (SC) in patients with strokes and brain injuries have been established. ${ }^{10,11}$ Scientistsrevealedthatintravenouslyorintraarteriallyadministered of SChave extremely low ability to penetrate through blood-brain barrier from blood stream to brain tissue. Administration of SC into cerebrospinal fluid is complicated by craniocaudal flow of liquor. Neurosurgeons inject SC directly into brain tissue, but these manipulations are accompanied with additional surgical intervention (skull trepanation) worsening patient's state. Perineural administration of mesenchymal stem cells (MSC) into brain appeared to bea kind of way out. ${ }^{11-13}$ Enhancement of this technique allowed using somatotopic principle of cranial nerves' organization for targeted migration of SC to specific brain region..$^{11,12,14}$ In particular, intranasal perineural SC injection mainly through the system of olfactory nerves can be used in case of stroke located in anterior or middle cranial fossa. Localization of destructive focus in posterior cranial fossa requires $\mathrm{SC}$ injection into the area of trigeminal nerve endings in inferior nasal concha or directly into Meckel's cave. The study was aimed at pilot clinical testing of SC perineural transport technique 
with attention to somatotopic principle of exogenous SC distribution in the area of brain injuries.

\section{Material and methods}

\section{Experimental stage}

Wistar rats $(n=40)$ weighing 210-250grams was subjected to brain tissue removal $(100 \mu \mathrm{l})$ by aspiration with micro pipettein a stereo taxic device under ketamine-xylazine-acepromazine anesthesia (55.6, 6.6 and $1.1 \mathrm{mg} / \mathrm{kg}$, respectively, $\mathrm{i} / \mathrm{p}$ ). Two groups of rats hadbilateral destructions in somatosensory $(n=20)$ and cerebellar $(n=20)$ cortices. MSCsuspension (30000 cells labelled by PKH67 green fluorescent linker(Sigma-Aldrich, St. Louis, MO, USA), in $50 \mu 1$ of phosphatebuffered saline) was injected into sub mucosa of nasal cavity or into Meckel's cave-in 10min after surgery. 10 rats from each group received microinjection of MSC into sub mucosa of nasal cavity (Figure 1), another 10 received microinjection of MSC into Meckel's cave (Figure 1). The method details are described. ${ }^{11-14}$ Animals were sacrificed in $0.5,1.0,4.0,8.0,24.0$ and 72.0hours and in 7, 14 and 21days after procedures. Brain was extracted, frozen and sliced for confocal laser microscopy (Zeiss AxioVert 200M fluorescent microscope with Zeiss Axio Cam HRm CCD camera, Carl Zeiss, Germany).
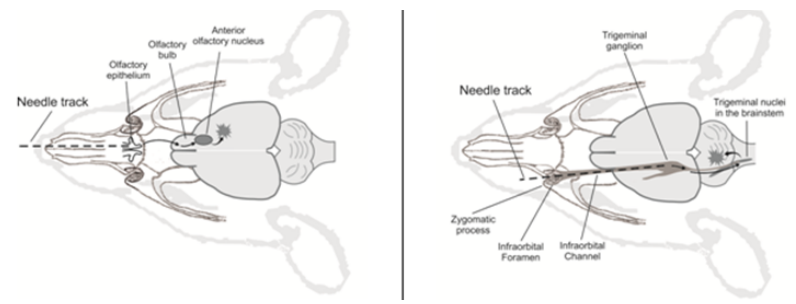

Figure I Scheme of stem cells injection in rat using intranasal way (left and infraorbital channel in to area of Gasser's ganglionin Meckel'scave (right).

Clinical stage early passages of cell cultures of autologous MSC from AT have been obtained from 23 patients with brain strokes aged $47.4(28.3 \div 65.0)$ at the stage of cell material preparation for implantation. Viability of cell cultures, proliferative potential, expression of standard and additional phenotypic MSC markers have been comparatively assessed. Concentration of gill cell-derived neuro trophic factor (GDNF) was estimated in MSC AT cultures of patients with brain strokes.

It was revealed that MSCAT cultures of patients with brain strokes correspond to main morpho-phenotypic criteria defined by International Society for Cellular Therapy, express nerve growth factor CD271, maintain proliferative potential and are characterized by spontaneous production of GDNF at early stages of cell cultures pass aging. Preparation was followed by three subsequent perineural endoscopic implantations of autologous MSC from adipose tissue in the amount of $5 \times 106$ up to $12 \times 106$ cells to 23 patients with $5-9$ days intervals.

\section{Results}

\section{Experimental stage}

PKH67 signal was observed in olfactory bulb in 0.5 hours and in somatosensory zone in 24.0 hours after intranasal application in rats with somatosensory cortex lesion with thepeak on 14th-21st days (Figure 2A). The signal was vague in rats with cerebellar cortex lesion (Figure 2B). Conversely, the signal was detected in rats with cerebellar cortex lesion in caudal brainstem in 4.0 and 8.0hours and in cerebellar cortex in 24.0hours after microinjection into Meckel's cave with the peak on $21^{\text {st }}$ day (Figure 2D). Microinjection into Meckel's cave was followed by weak signal in the damaged area in animals with somatosensory cortex lesion (Figure 2C). Our data demonstrate targeted migration of mesenchymal stem cells towards brain tissue of
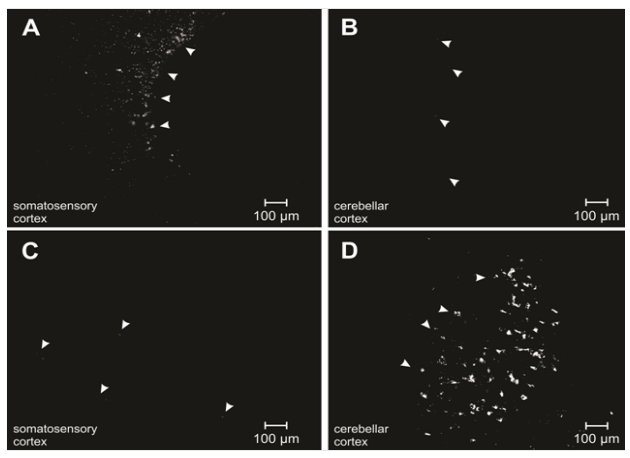

anterior and posterior cranial fossa depending on the way of delivery (olfactory or trigeminal routes).

Figure 2 Fluorescent photographs showing the PKH67 labelled cells on 2 Istday after surgery in the lesioned brain areas contralateral to microinjection site. $A$ and $B$ following intranasal microinjection, $C$ and $D$ following microinjection into Meckel's cave.

\section{Clinical stage}

Good to lerability of cell therapy and absence of toxicreactions and other side effects mwere registered in all 23 cases of perineural injection of MSC AT. Stable and pronounced recovery of neurological functions was noted during first 24hours after each MSC injection, and it preserved in future. Initial assessment of neurologic deficiency according to NIHSS scale ${ }^{15}$ in 14 patients with initial cerebral infarctions who received both perineural MSC AT and ongoing standard therapy was 10.1 points and 1.9 points in 6months; no one had re-infarction. Initial assessment of neurologic deficiency according to NIHSS scale in 9patients with multiple secondary cerebral infarctions after old intracranial hemorrhages was 27.8 points and 14.2 points in 6 months after standard therapy combined with perineural injection of MSC AT.

Initial assessment of neurologic deficiency according to NIHSS scale in 7 patients who received only standard therapy was 11.6 points and 10.2 points in 6 months; two of them $(28.6 \%)$ had cerebral reinfarctions.

\section{Conclusion}

Therefore, cell therapy with autologous MSC from AT by means of perineural delivery to brain has pronounced positive effect on recovery of neurological functions in patients with brain attacks. This effect is based on the principle of somatotopic migration of SC along cranial nerve fibers to brain injuries in specific regions. Less traumatic delivery of exogenous autologous MSC to injured brain regions contribute to activation of reparative processes in nerve tissue and effective recovery of neurological functions in patients with cerebral infarctions.

\section{Acknowledgements}

This pooled analysis was funded by SSTP "New methods of 
medical care", section "Transplantation of cells, tissues and organs" (2016-2020), and by grant OOO "Synergy".

\section{Conflict of interest}

All listed authors concur with the submission of the manuscript; all authors have approved the final version. The authors have no financial or personal conflicts of interest.

\section{References}

1. Chen S, Singh RJ, Kamal N, et al. Improving care for acute in-hospital ischemic strokes-A narrative review. Int $J$ Stroke. 2018; 1:1747493018790029.

2. Lioutas VA, Marchina S, Caplan LR, et al. Endotracheal intubation and in-hospital mortality after intracerebral hemorrhage. Cerebrovasc Dis 2018;45(5-6):270-278.

3. ChungJW, ParkSH, KimN, et al. Trial of ORG 10172 in Acute Stroke Treatment (TOAST) classification and vascular territory of ischemic stroke lesions diagnosed by diffusion-weighted imaging. J Am Heart Assoc. 2014;3(4):pii:e001119.

4. Doeppner TR, Hermann DM. Stem cell-based treatments agains stroke: observations from human proof-of-concept studies and considerations regarding clinical applicability. Front Cel lNeurosci. 2014;8:357.

5. Doeppner TR, Hermann DM. Editorial: Stem cells and progenitor cells in ischemic stroke-fashion or future? Front Cell Neurosci. 2015;9:334.

6. Doeppner TR, Bähr M, Hermann DM, et al. Concise review: extracellular vesicles overcoming limitations of cell therapies in ischemic stroke. Stem Cells Transl Med. 2017;6(11):2044-2052.
7. Sharma A. Stem cell research and policy in India: current scenario and future perspective. J Stem Cells. 2009;4(2):133-140.

8. Kalladka D, Sinden J, Pollock K, et al. Human neural stem cells in patients with chronic ischaemic stroke (PISCES): a phase 1, first-inman study. Lancet. 2016;388(10046):787-796.

9. Qiao LY, Huang FJ, Zhao M, et al. A two-year follow-up study of cotransplantation with neural stem/progenitor cells and mesenchymal stromal cells in ischemic stroke patients. Cell Transplant. 2014;23 Suppl 1:S65-S72.

10. Xiao J, Nan Z, Motooka Y, et al. Transplantation of a novel cell line population of umbilical cord blood stem cells ameliorates neurological deficits associated with ischemic brain injury. Stem Cells Dev. 2005;14(6):722-733.

11. Kulchitsky V, Zamaro A, Shanko Y, et al. Positive and negative aspects of cell technologies in cerebral diseases. J Neurol Stroke. 2018;8(2):87-88.

12. Donega $\mathrm{V}$, Nijboer $\mathrm{CH}$, Braccioli $\mathrm{L}$, et al. Intranasal administration of human MSC for ischemic brain injury in the mouse: in vitro and in vivo neuroregenerative functions. PLoS One. 2014;9(11):e112339.

13. Kulchitsky V, Zamaro A, Navitskaya V, et al. Perspectives of stem cells use in alzheimer's disease treatment. J Neurol Stroke. 2018;8(3):190 191.

14. Roch M, Messlinger K, Kulchitsky V, et al. Ongoing activity in trigeminal wide-dynamic range neurons is driven from the periphery. Neuroscience. 2007;150(3):681-691.

15. Jeyaseelan RD, Vargo MM, Chae J. National institutes of health stroke scale (NIHSS) as an early predictor of poststroke dysphagia. PMR. 2015;7(6):593-598. 